\title{
CLINICAL CASE OF A CHILD WITH RHABDO- MYOSARCOMA (RMS), EMBRYONIC TYPE, WITH ORAL COMPLICATIONS POST RADIATION THERAPY AND CHEMOTHERAPY
}

\author{
M. Rashkova, V. Alexieva, G. Jegova \\ Department of Pediatric Dentistry \\ Faculty of Dental Medicine, Medical University - Sofia, Bulgaria
}

\section{SUMMARY}

Case of a 7 years old female, with significant medical history of embrional type of RMS, diagnosed 2,5 years ago in left parapharingeal area. After surgical removal of the tumor, the area is radiated with 50Gy for 6 weeks and also has 3 courses of chemotherapy. By now the child is clinically healthy of RMS, but she has developed serious side effects of radiation therapy: erosive caries to all primary teeth, hyposalivation and total lost of function of the left parotid gland, delayed development of the teeth, facial asymmetry, caused by the arrested growth of left side mandibule.

Acute and chronic complications of radiation therapy act to the dynamic changes of the oral environment in a phase of active growth, dental eruptions and incomplete mineralization. The potential risk of osteoradionecrosis and radiation caries, resulted in the sequel of xerostomia and a cariogenic shift in micro flora, requires a prophylactic and heeling program to modulate the oral environment and to prevent or at lease, minimize the side effects and should be integral part of the treatment. Frequent dental follow - up should be scheduled throughout the treatment period to reinforce the importance of continued oral hygiene and to deal with the complications for a lifelong period of time.

Key word: Rhabdomyosarcoma (RMS) embryonic, alveolar RMS, and pleomorphic RMS, chemotherapy, radiation therapy, risk- oral environment, radiation caries, osteoradionecroses, salivary flow.

\section{INTRODUCTION}

Rhabdomyosarcoma (RMS) is the most common soft-tissue sarcoma of childhood and adolescences, involving the head and neck. RMS can be described by three basic types: embryonic, alveolar and pleomorphic. Embryonic type is occurring in about $60 \%$ of the cases and appears within the first 10 years of life. Treatment approaches to RMS incorporates surgery, radiation and chemotherapy, increase the chances of this malignant desease heeling $(1,2,3,4)$.
Complications that may develop in oral tissues and craniofacial skeleton growth in result of treatment can cause severe disturbances in the dynamic age of growth $(5,6,7$, 9 ). They can be minimized by special preventive programs applied before, during and after the radiation therapy. In the long-term care of the irradiated child should be frequent dental follow - up to deal with complications of risky oral environment and preventing the oral pathology $(10,11,12)$.

\section{Case presentation}

We present the case of a 7 years old girl with significant medical history of rhabdomyosarcoma, embryonic type, diagnosed 2.5 years ago in the left parapharangeal area. After surgical removal of the tumor, the area was radiated with $50 \mathrm{GY}$ for six weeks and passed three courses of chemotherapy.

By now the child is clinically healthy regarding RMS, but has developed serious side effects due to radiation and chemotherapy:

\section{Extraoral status}

In extraoral status shows visible facial asymmetry with deviation of lower jaw to the left.There also is a visible cicatrix in result of the surgical treatment and a clear dysfunction symptomatics of left $n$.facialis: inability to close lower left eyelid and left mouth angle drop.Occlusion enters the standard, but when mouth is opening shows a deviation of the middle line, caused by arrested bone growth and secondary effect of radiation on cartilaginous growth centers located in the condyls of the mandible. 


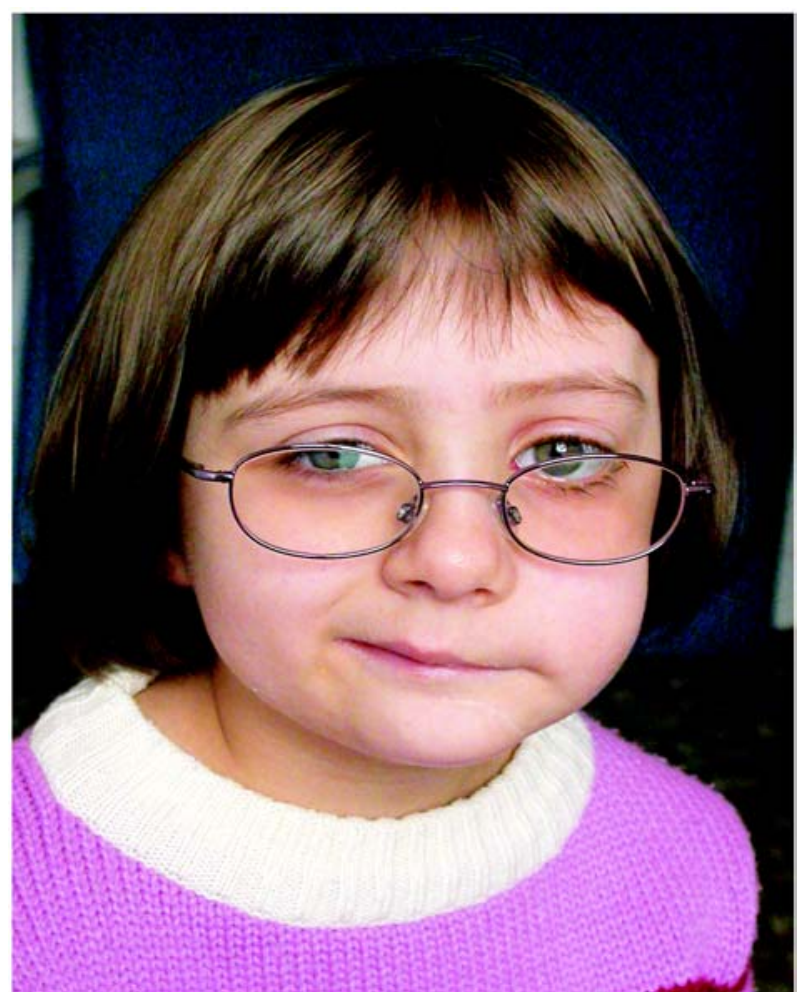

Image 1.

\section{Oral cavity risk assessment}

The child is subjected to clinical oral examination and evaluation of oral medium and dental caries:

- Dental caries risk assessment(8);

- Registration of the dental status;

- Evaluation of the saliva through "Saliva Check" GC test .

- Evaluation of the oral microorganisms(CRTtest, Vivadent).

\subsection{Registration of the dental status}

Delayed dental eruption is observed. At high radiation doses there may occur disturbances in odontogenesis. (Mother of the child refuses orthopantomographia).

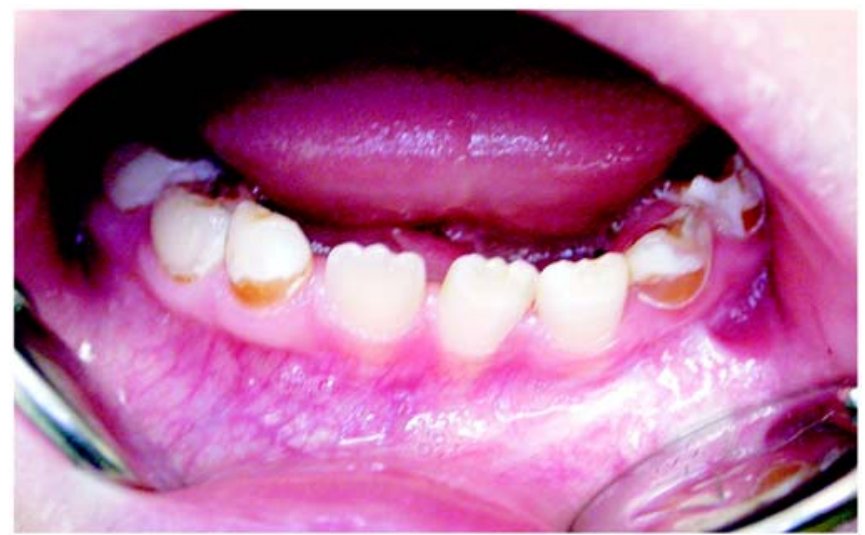

Image 2.

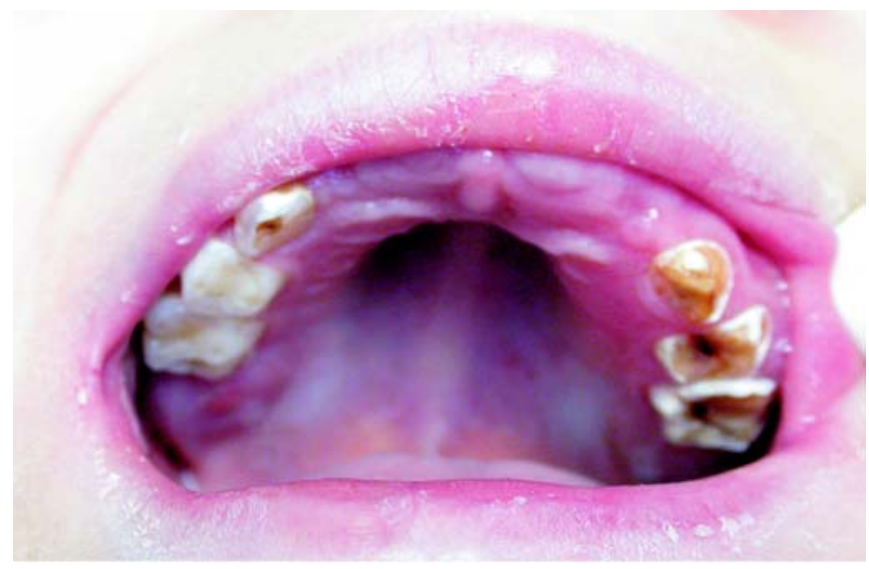

Image 3.

Primary teeth have lesions typical for radiation caries. In the left side, where irradiation was focused, there are dematerialized lesions typical for radiation carries on no predilection sides of dental crowns. Lesions are spread all over the teeth - fissures, proximal contacts and occlusal areas.

Lower primary teeth in the right-hand side have reduced caries lesions in the area of proximal contacts and fissures. These molars are not diffusely destroyed in the area of occlusion. gingival.

There are no visible changes to lips, mucosa and

2.2. Evaluation of the saliva through "Saliva Check" GC test:

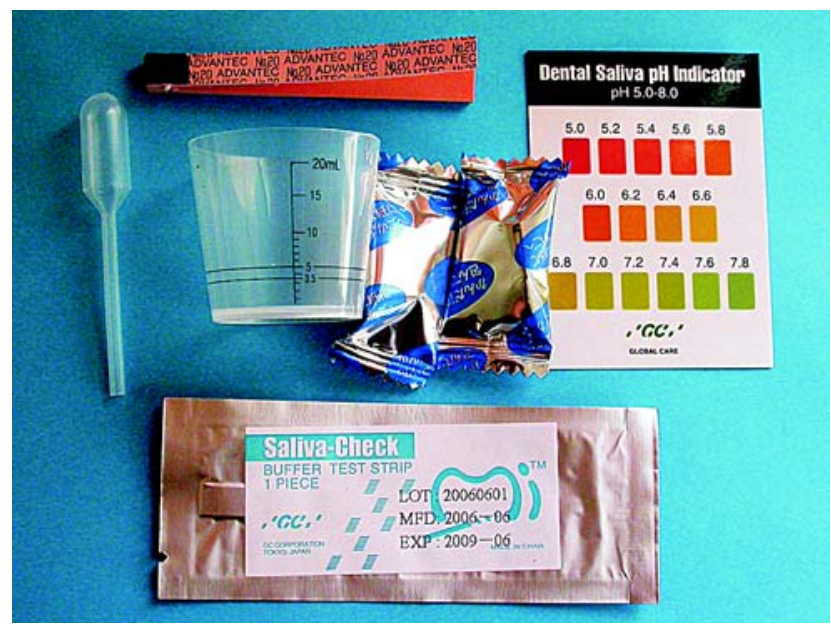

- Check up of a non-stimulated salivary flow shows preserved function of small salivary glands;

- Check up of a stimulated salivary flow - after chewing a piece of wax gum for $30 \mathrm{sec}$., the produced saliva was collected in a cup for $5 \mathrm{~min}$. The quantity of the collected saliva was $1.5 \mathrm{ml}$, demonstrating strongly decreased salivary secretion; 
- The saliva consistence contains bubbles corresponding to code II.

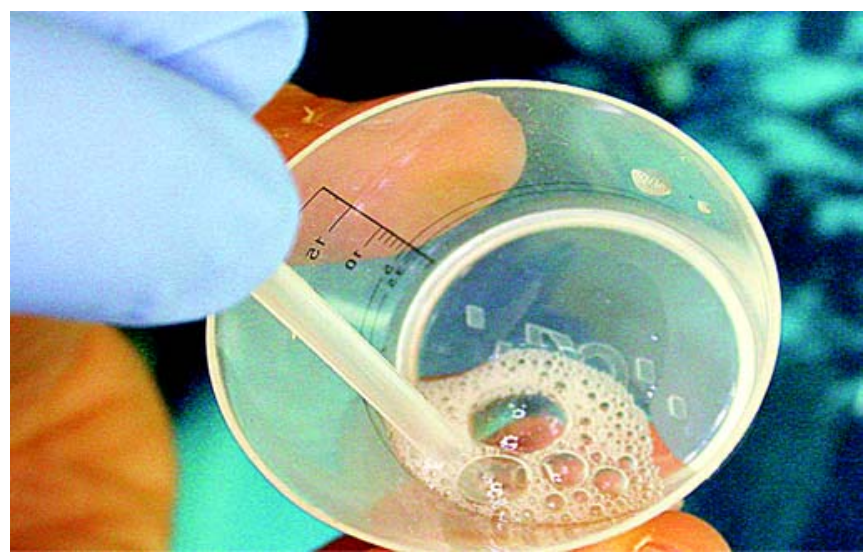

- Check up of $\mathrm{pH}$ of the saliva, with a lacmus testtape, laid on the back of the tongue for a few seconds, shows pH 6, 8, which is a lower degree of normal saliva;

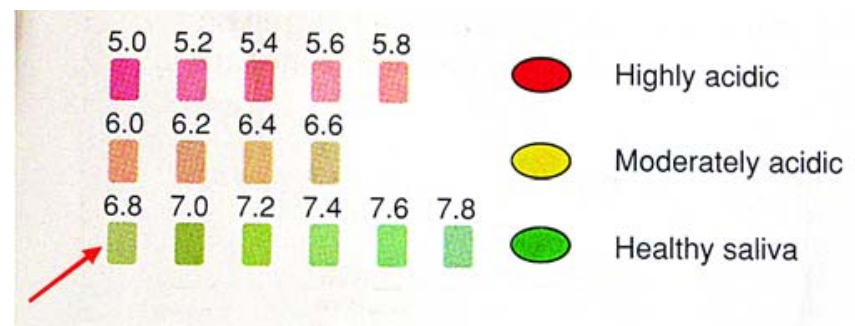

- Check up of saliva buffer capacity - slightly decreased ( 9 points);

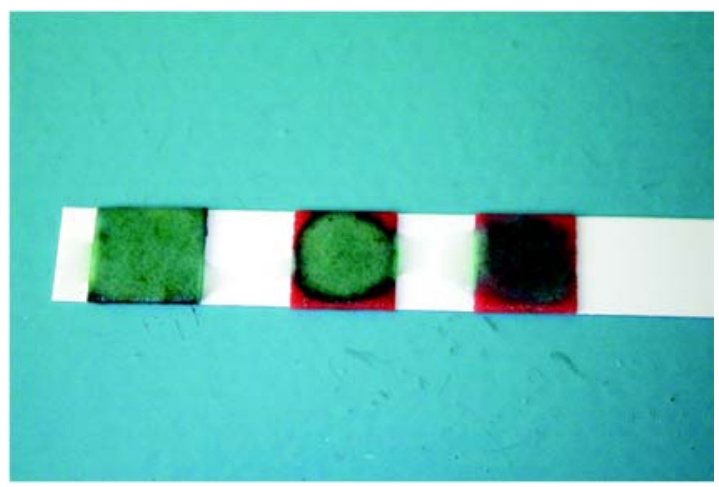

\subsection{Evaluation of cariogenic microorganisms:}

CRT ( Vivadent) - microbiological test was used to determine the quantity of Mutans streptococci and Lactobacilli. After a cultivation in thermostat for $48 \mathrm{~h}$, reported growth of M. Streptococci and Lactobacilli $<105$.

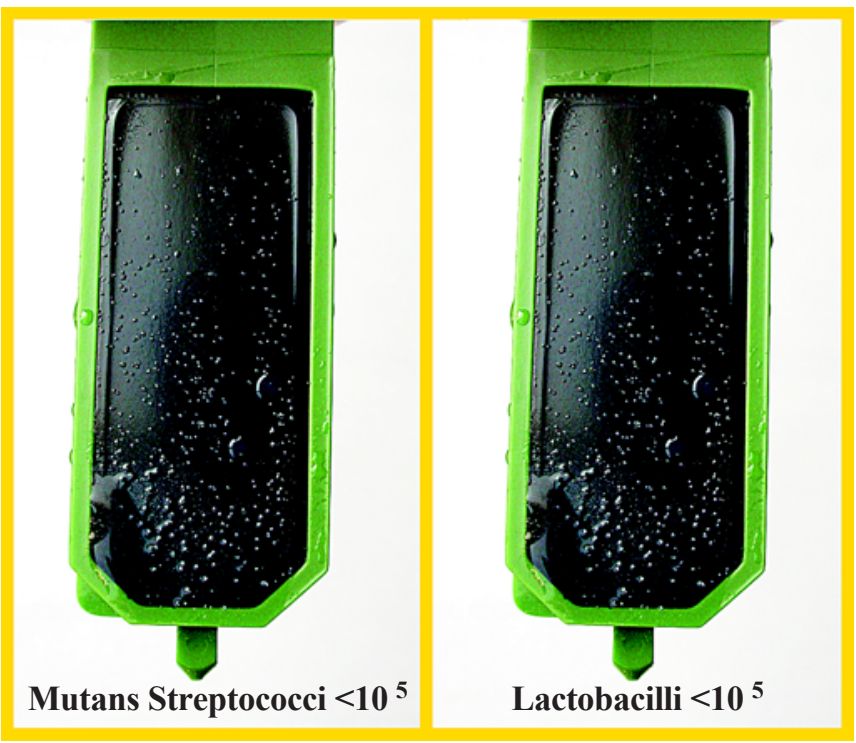

Conclusion of the clinical observation:

Observed complications after irradiation: radiation dental caries to the primary teeth, high risky oral environment, arrested or decreased eruption of teeth and growth of the mandible in left side, as well as difficult chewing are leading factors for elaboration of a specific program to heel or prevent these side effects.

Complication as osteoradionecroses, which might occur in the period of dental eruption, needs constant care to maintain an oral environment in law rates of risk and high rate of oral protecting factors.

We offer the following preventive-healing program for this particular case and this program can be used for children, which were treated with radiation in the head area.

Program for prevention and treatment of children with oral complications post radiation therapy and chemotherapy

- Making parents familiar with occurring or possible future complications due to irradiation and participation of parents in forthcoming treatment, prevention and control;

- Oral environment modeling program:

> Correction of reduced salivation by artificial saliva; $>$ motivation and training for proper oral hygiene;

> maintenance of low microbe number: gargling by chlorhexidine solution in the period of tooth eruption;

> maintenance of optimum ion balance and buffer capacity - daily application of remineralizing foam - Tooth Mousse GC;

> local fluorine applications:

$\gg$ twice a year Fluor- varnish;

» once in 2 months professional application of F-

gel;

» daily application of F-tooth paste;

> Transition toward regime of optimal caries-resist 
feeding.

- Professional oral hygiene once in three months;

- Control check ups at intervals of two months especially until tooth eruption completes;

- Antibiotic premedication at necessity of extraction;

- Periodical check up of saliva and oral microflora;

- Radiation caries heeling:

> Temporalization of all radiation lesions until complete heeling with the purpose to regulate oral micro flora;

> Dinamic fluor- treatment by glass ionomer cement;

> More radical approach to complicated caries due to expected damages in pulp resulted by radiation therapy;
> Prophylactic and therapeutic silanisation; preventive obturation;

- Prevention and heeling of expected tooth anomalies of permanent teeth after their eruption.

\section{CONCLUSION:}

Irradiation in childhood leads to severe oral complications continuing in time and demanding special training of both parents and paediatric dentist. Prevention or reduction of such lateral effects is possible and should be started before, during and after irradiation. It has to become integral part of heeling head and neck tumors.

\section{REFERENCES:}

1. Paulino A. C, J. H. Simon, W. Zhen and B. C. Wen. Long-term effects in children treated with radiotherapy for head and neck rhabdomyosarcoma, Int J Radiat Oncol Biol Phys 2000, 48,1489-1495.

2. Burket's Oral Medicine ed. Greenberg . Glick - Lester William Burket, Martin S. Greenberg, Michael Glick 248255.

3. Hancock PJ, Epstein JB, Sadler GR. Oral and dental management related to radiation therapy for head and neck cancer. J Can Dent Assoc 2003; 69(9):585-90.

4. J. A. Toljanic, R. H. Heshmati and J. F. Bedard, Dental follow-up compliance in a population of irradiated head and neck cancer patients, Oral Surg Oral Med Oral Pathol Oral Radiol Endod 2002,(93),35-38.
5. Kostler W. J., Hejna M., Wenzel C., Zielinski C. C. Oral mucositis complicating chemotherapy and/or radiotherapy: options for prevention and treatment. CA Cancer J Clin 2001; 51 (5): 290-3158.

6. Moller and M. Perrier, Dentomaxillofacial sequelae in a child treated for a rhabdomyosarcoma in the head and neck: a case report, Oral Surg Oral Med Oral Pathol 1998, (86) 297-303.

7. Naima Otmani Oral and Maxillofacial Side Effects of Radiation Therapy on Children, JCDA - www.cdaadc.ca/jcda,April 2007, Vol. 73, No. 3.

8. Rashkova M., M. Peneva, L. Doychinova, Study of the risk factors for the development of dental caries and creation of a system for assessment the risk of caries in children in Bulgaria OHDMBSC,2008, 7,(2) - June,3-11.

9. S. Y. Chen, A. Thakur, A. S. Miller and R. D. Harwick, Rhabdomyosarcoma of the oral cavity. Report of four cases, Oral Surg Oral Med Oral Pathol Oral Radiol Endod 1995(80), 192-201.

10. Scully C, Sonis S, Diz PD. Oral mucositis. Oral Dis 2006; 12(3):229-41.

11. Tenovuo J. O. Salivary parameters of relevance for assessing caries activity in individuals and populations. Comm Dent Oral Epidemiol 1997;25(8)82-6.

12. Vissink A., Jansma J., Spijkervet F.K., Burlage F.R., Coppes R.P. Oral sequelae of head and neck radiotherapy. Crit Rev Oral Biol Med 2003; 14(3):199212.9.

\author{
Address for correspondence: \\ Maya Rashkova, Associate professor, DDS, Ph.D \\ Department of Pediatric Dentistry, \\ Faculty of Dental medicine, Medical University - Sofia, \\ 1, G. Sofiyski Str., Sofia 1000, Bulgaria. \\ Mobile: 0359888215033 \\ e-mail: mayarashkova@mail.bg
}

